\title{
Increased 18F-FDG uptake mimicking thyroid cancer in a patient with Hashimoto's thyroiditis
}

\author{
Schmid, Daniel T ; Kneifel, Stefan ; Stoeckli, Sandro J ; Padberg, Barbara-C ; Merrill, Griff ; Goerres,
} Gerhard W

\begin{abstract}
We report the case of a 68-year-old patient with a known paravertebral malignant schwannoma, sent to us for postoperative staging. A combined whole-body PET/CT scan showed only poor $18 \mathrm{~F}$-fluorodeoxyglucose uptake in the region of the primary tumor but distinct increased fluorodeoxyglucose uptake in the left and right thyroid gland. Thyroid sonography showed two hypoechogenic nodules. Ultrasound-guided fine-needle aspiration biopsy of one nodule showed oxyphil transformed cells, compatible with malignancy. Based on these findings, the patient underwent a subtotal thyroidectomy. Histopathology of the specimen revealed a chronic follicular Hashimoto's thyroiditis. This case demonstrates that Hashimoto's thyroiditis can mimic thyroid cancer in PET but also in sonography and fineneedle aspiration biopsy
\end{abstract}

DOI: https://doi.org/10.1007/s00330-002-1619-4

Posted at the Zurich Open Repository and Archive, University of Zurich

ZORA URL: https://doi.org/10.5167/uzh-156130

Journal Article

Published Version

Originally published at:

Schmid, Daniel T; Kneifel, Stefan; Stoeckli, Sandro J; Padberg, Barbara-C; Merrill, Griff; Goerres, Gerhard W (2003). Increased 18F-FDG uptake mimicking thyroid cancer in a patient with Hashimoto's thyroiditis. European Radiology, 13(9):2119-2121.

DOI: https://doi.org/10.1007/s00330-002-1619-4 


\author{
Daniel T. Schmid \\ Stefan Kneifel \\ Sandro J. Stoeckli \\ Barbara-C. Padberg \\ Griff Merrill \\ Gerhard W. Goerres
}

\section{Increased 18F-FDG uptake mimicking thyroid cancer in a patient with Hashimoto's thyroiditis}

Received: 18 April 2002

Accepted: 4 June 2002

Published online: 30 August 2002

(C) Springer-Verlag 2002

D.T. Schmid ( $)$ S. Kneifel · G. Merrill

G.W. Goerres

Division of Nuclear Medicine,

University Hospital Zurich,

Raemistrasse 100, 8091 Zurich,

Switzerland

e-mail: daniel.schmid@dmr.usz.ch

Tel.: +41-1-2553555

Fax: +41-1-2554414

\section{S.J. Stoeckli}

Clinic of Otolaryngology,

Head and Neck Surgery,

University Hospital Zurich,

Raemistrasse 100, 8091 Zurich,

Switzerland

Barbara-C. Padberg

Department of Pathology,

University Hospital Zurich,

Raemistrasse 100, 8091 Zurich,

Switzerland

\begin{abstract}
We report the case of a 68-year-old patient with a known paravertebral malignant schwannoma, sent to us for postoperative staging. A combined whole-body PET/CT scan showed only poor ${ }^{18} \mathrm{~F}$-fluorodeoxyglucose uptake in the region of the primary tumor but distinct increased fluorodeoxyglucose uptake in the left and right thyroid gland. Thyroid sonography showed two hypoechogenic nodules. Ultrasoundguided fine-needle aspiration biopsy of one nodule showed oxyphil transformed cells, compatible with malignancy. Based on these findings, the patient underwent a subtotal thyroidectomy. Histopathology of the specimen revealed a chronic follicular Hashimoto's thyroiditis. This case demonstrates that Hashimoto's thyroiditis can mimic thyroid cancer
\end{abstract}

in PET but also in sonography and fine-needle aspiration biopsy.

Keywords PET $\cdot$ Hashimoto's thyroiditis · Oncocytic lesion . Pitfalls

\section{Introduction}

Fluorodeoxyglucose/positron emission tomography (FDGPET) can detect incidental thyroid lesions in approximately $2 \%$ of patients with no known thyroid disease [1]. Forty-seven percent of the time incidental FDG uptake in the thyroid is caused by malignancy [1]; therefore, such lesions must be examined minutely. Because both tumor and inflammatory lesions can show increased FDG uptake, other diagnostic modalities are needed to confirm the findings [2]. Diffuse FDG uptake can be an indicator of chronic thyroiditis and sonography often facilitates the diagnosis in these patients [2].

\section{Case report}

A combined whole-body PET/CT scan was performed in a 68-year-old woman for the planning of conformal radiotherapy of the paravertebral region where previously a malignant schwannoma was incompletely resected (R1). This scan was obtained with an in-line PET/CT scanner (Discovery LS, GE Medical Systems, Waukesha, WI) which allows the acquisition of hardware co-registered CT and PET images in one session. Postoperatively only poor FDG uptake was found in the region of the primary tumor but increased FDG uptake in the left and right thyroid gland. Automatic co-registration of CT and PET data revealed two well-circumscribed lesions within the left and right lobe of the thyroid gland (Fig. 1). The patient showed no clinical signs of thyroid insufficiency (TSH, T3, and T4 levels were normal). Neither an inflammatory disease nor any signs suspicious for thyroid cancer 
Fig. 1 A Computed tomography, B positron emission tomography (PET), and $\mathbf{C}$ fusion image showing two nodules (arrowheads) within the thyroid gland with increased fluorodeoxyglucose (FDG) uptake in PET
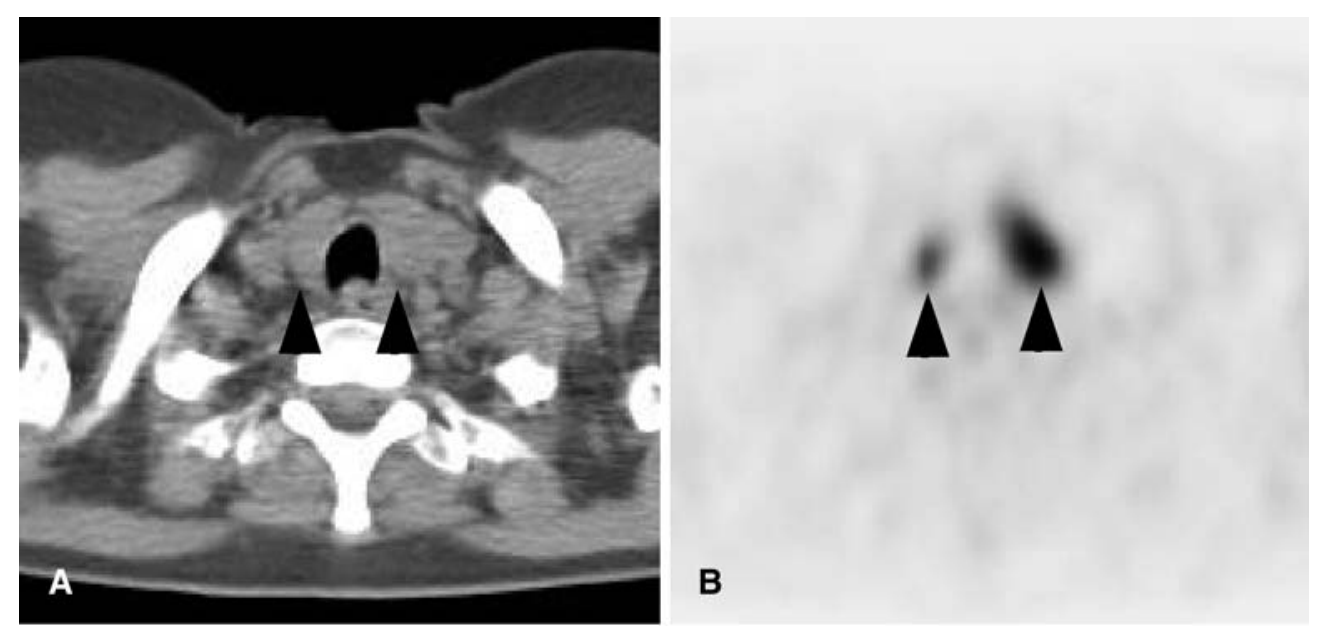

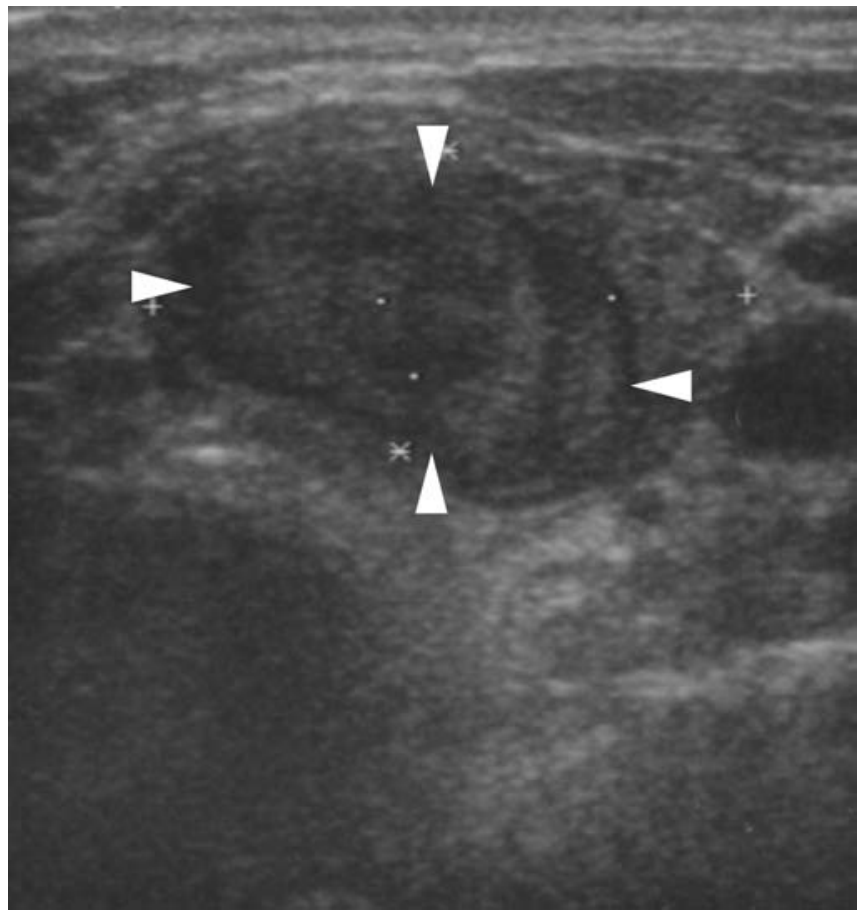

were noted. The autoimmune antibodies were not determined. Ultrasound showed two irregularly shaped hypoechogenic nodules in the thyroid, one in each lobe of the gland $(6 \times 6 \times 11$ and $20 \times 12 \times 21 \mathrm{~mm}$ ), both not palpable upon clinical examination (Fig. 2)

An ultrasound-guided fine-needle aspiration biopsy was taken from the larger nodule. Clusters of Hurthle cells (oxyphilic follicular epithelium) with only a few scattered lymphocytes were found microscopically (Fig. 3A). The patient underwent surgery for possible oncocytic thyroid carcinoma. The definitive histology of the nodules revealed the typical histologic features of Hashimoto's thyroiditis with an admixture of lymphocytes, plasma cells, cells representing the germinal center of lymphoid follicles, and predominant oxyphilic follicular epithelium (Fig. 3B).

\section{Discussion}

This case demonstrates how chronic thyroiditis can mimic thyroid cancer in FDG-PET but also in sonography and fine-needle aspiration biopsy. An inflammatory disease should always be part of the differential diagno-

Fig. 2 Sonography of the left thyroid gland showing a hypoechogenic nodule $(20 \times 12 \times 21 \mathrm{~mm}$; arrowheads $))$ 


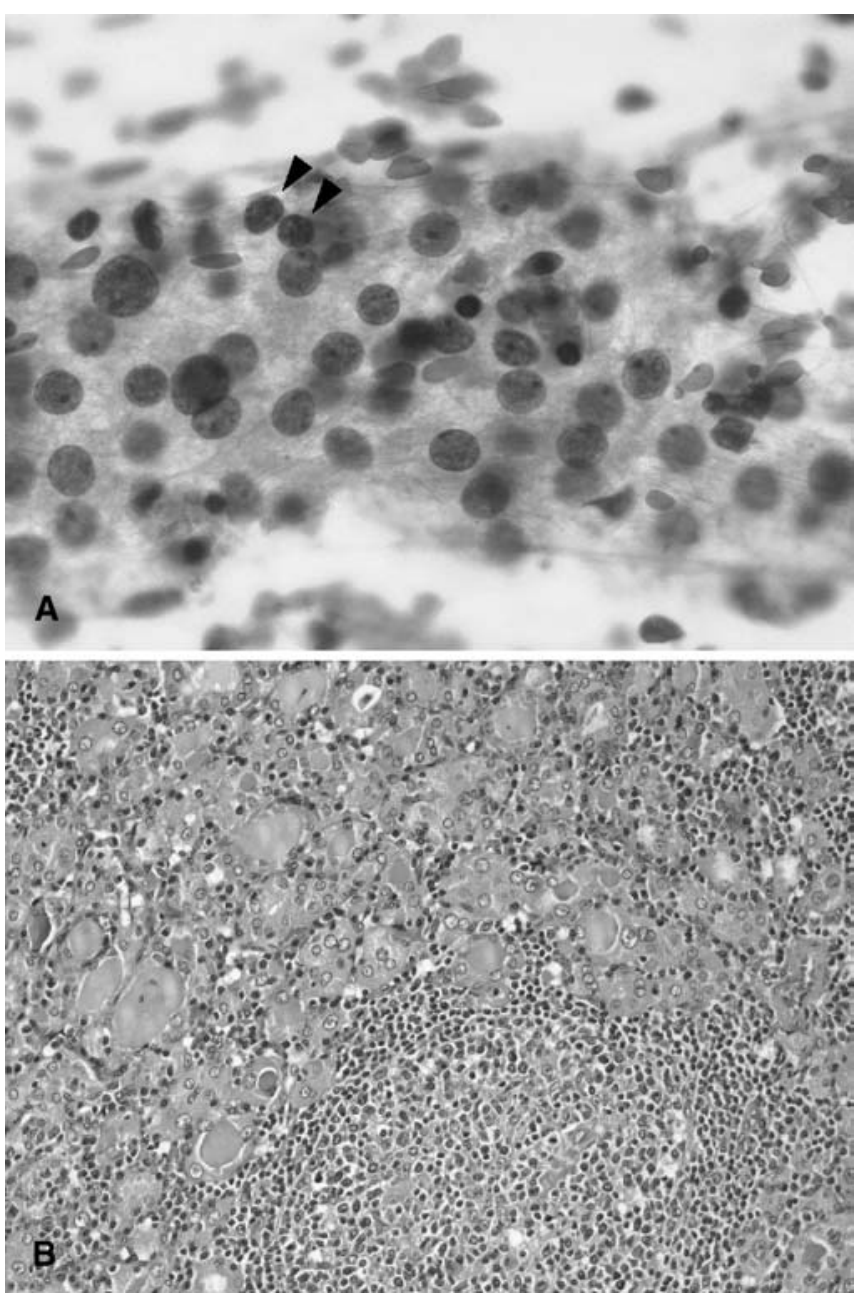

sis in patients with diffuse FDG uptake of the thyroid gland [2]. Using PET/CT, increased FDG uptake could be assigned to well-circumscribed nodules in the thyroid gland. This pattern of FDG uptake has not yet been described in Hashimoto's thyroiditis. Increased focal FDG uptake has also been described in a patient with a Hurthle cell tumor (oncozytoma) [3]. In this context it can be suggested that increased FDG uptake can be based on oncocytic transformed cells in Hashimoto's thyroiditis and Hurthle cell tumors or lymphocyte proliferation as verified histologically in our case. Lymphocyte proliferation can cause increased FDG uptake as described in a patient with chronic tonsillitis [4]. The growing number of PET examinations will likely lead to an increased number of incidental thyroid lesions in patients without clinical symptoms. As illustrated in this case, the number of patients receiving surgical treatment for suspicious lesions might also increase.

Fig. 3 A Papanicolaou (magnification, $\times 500$ ). Cytologic features: group of Hurthle cells with only a few scattered lymphocytes (arrowheads). B Hematoxylin-eosin enlargement (magnification, $\times 120$ ). Histology of Hashimoto's thyroiditis: lymphocytic infiltration of the fully transformed oxyphilic follicular epithelium

\section{References}

1. Cohen MS, Arslan N, Dehdashti F et al. (2001) Risk of malignancy in thyroid incidentalomas identified by fluorodeoxyglucose-positron emission tomography. Surgery 130:941-946
2. Yasuda SA, Shohtsu et al. (1998) Chronic thyroiditis: diffuse uptake of FDG at PET. Radiology 207:775-778

3. Wiesner W, Engel H, Schulthess GK von et al. (1999) FDG-PET-negative liver metastases of a malignant melanoma and FDG-PET positive Hurthle cell tumor of the thyroid. Eur Radiol 9:975-978
4. Kawabe J, Okamura T, Shakudo M et al. (1999) Two cases of chronic tonsillitis studied by FDG-PET. Ann Nucl Med $13: 277-279$ 